\title{
Análise do treinamento por reforçamento positivo para habituação de Sapajus apella em aparato experimental
}

\section{Analysis of positive reinforcement training for habituation of Sapajus apella in experimental apparatus}

\author{
Rafaela Martins Reis ${ }^{1,2}$ (D), Leon Claudio Pinheiro Leal1,2 (iD), José Augusto Pereira Carneiro Muniz (iD, Carlomagno \\ Pacheco Bahia ${ }^{1}$, Lane Viana Krejcová ${ }^{1,2}$ iD \\ ' Universidade Federal do Pará, Instituto de Ciências e da Saúde, Laboratório de Neuroplasticidade, Belém, Pará, Brasil \\ ${ }^{2}$ Centro Nacional de Primatas, Ananindeva, Pará, Brasil
}

\begin{abstract}
RESUMO
OBJETIVO: Determinar o padrão de aprendizagem e as diferenças inter e intraindividuais observadas em treinamento para entrada voluntária e posicionamento de macacos Sapajus apella em aparato experimental (cadeira para primatas), bem como quantificar o tempo necessário para a obtenção dos resultados esperados. MATERIAIS E MÉTODOS: Participaram da pesquisa três macacos machos que foram submetidos a um período de dois meses de habituação aos experimentadores, seguido de sessões diárias de 20 min de treinamento por condicionamento operante, com o emprego das técnicas de clicker e reforçamento positivo por meio de recompensas comestíveis, pelo período de quatro meses. As sessões foram gravadas para posterior análise de parâmetros temporais e de frequência de apresentação dos comportamentos desejados. RESULTADOS: Análises interindividuais revelaram diferenças significativas na comparação dos escores obtidos para cada animal nas latências e frequências de apresentação dos comportamentos desejados. Análises intraindividuais comparando diferentes janelas temporais ao longo do período de treinamento revelaram diferenças significativas de desempenho dos animais nos diferentes períodos, refletidos nas curvas de aprendizado individuais que demonstraram alcance de platô para diferentes parâmetros comportamentais para cada animal. CONCLUSÃO: O reforço positivo se mostrou eficaz no treinamento para posicionamento na cadeira para primatas. Possivelmente, fatores individuais influenciaram de forma significativa os padrões de aprendizagem. A aplicação de técnicas de reforçamento positivo influencia positivamente a relação humano-primata e o bem-estar desses animais experimentais, apesar da necessidade de disposição de tempo para o alcance dos padrões comportamentais desejados, constituindo assim uma importante técnica de refinamento e manejo de primatas.
\end{abstract}

Palavras-chave: Primatas; Bem-Estar do Animal; Comportamento Animal; Experimentação Animal; Reforço Positivo.

\begin{abstract}
OBJECTIVE: To determine the learning pattern and the inter and intraindividual differences observed in training for voluntary entry and positioning of Sapajus apella monkeys in an experimental apparatus (chair for primates), as well as to quantify the necessary time to obtain the expected results. MATERIALS AND METHODS: Three male monkeys underwent a two months period of habituation to the experimenters, followed by daily operant conditioning training sessions using the clicker technique and positive reinforcement through edible rewards, with daily training sessions of 20 min for a period of four months. The sessions were recorded for later analysis of temporal parameters and frequency of presentation of the desired behaviors. RESULTS: Interindividual analysis revealed significant differences in the comparison of the obtained scores for each animal in the latencies and frequencies of presentation of the desired behaviors. Intraindividual analysis comparing different time windows over the training period revealed significant contrast in the animals' performances within distinct periods, reflected in the individual learning curves that reached a plateau for different behavioral parameters for each animal. CONCLUSION: The use of positive reinforcement proved to be effective in training for positioning in the chair for primates. Possibly, individual factors significantly influenced learning patterns. The application of positive reinforcement techniques positively affects the human-primate relationship, influences the well-being of animals and, despite the need for time to reach the desired behavioral patterns, it is an important technique of refinement and animal management.
\end{abstract}

Keywords: Primates; Animal Welfare; Animal Behavior; Animal Experimentation; Positive Reinforcement.

\author{
Correspondência / Correspondence: \\ Lane Viana Krejcová \\ Universidade Federal do Pará, Instituto de Ciências da Saúde \\ Av. Generalíssimo Deodoro, 01. Bairro: Umarizal. CEP: 66050-160 - Belém, Pará, Brasil - Tel.: +55 (91) 98899-5477 \\ E-mail: lanekrejcova@gmail.com
}




\section{INTRODUÇÃO}

A importância do treinamento de animais como uma ferramenta útil para o cuidado e manejo em cativeiro vem sendo crescentemente reconhecida. Treinar animais para cooperar, voluntariamente, em procedimentos veterinários, de manejo ou de pesquisa, pode contribuir para melhorar a saúde e o potencial reprodutivo dos mesmos, diminuindo a necessidade de sedação e reduzindo o risco de ferimentos, com benefícios extensivos aos animais e a toda a equipe envolvida em seus cuidados, manutenção e pesquisa. Ainda, o treinamento oferece estímulo mental aos animais, expansão de repertórios comportamentais e aumento da atividade física. Animais treinados mantêm um alto grau de confiabilidade ao participar de procedimentos experimentais e de manejo e ficam menos estressados ao fazê-lo $\mathrm{o}^{1,2}$.

Dentre as abordagens que podem ser utilizadas para o treinamento, o reforçamento positivo figura como a alternativa mais recomendável, uma vez que fornece aos animais maiores escolhas e maior controle sobre suas vidas, o que pode contribuir para um bom estado psicológico e redução do estresse ${ }^{2}$. No reforçamento positivo, sempre que o animal faz o que o treinador deseja, ele recebe algo de que gosta como recompensa, o que induz a um aumento de apresentação dos comportamentos desejados. Essa técnica tem sido utilizada com sucesso no treinamento de animais em diferentes cenários, como zoológicos, criadouros e biotérios, para auxílio no manejo, no aprendizado de protocolos de pesquisa, em cuidados veterinários e como estratégia de enriquecimento ambienta| ${ }^{2,3,4}$

Primatas não humanos (doravante denominados primatas) estão entre os animais mais extensivamente estudados nos campos da pesquisa comportamental, psicologia, evolução, ecologia, conservação e antropologia, e são sujeitos experimentais de valor inestimável em pesquisas biomédicas devido às suas similaridades fisiológicas, genômicas, neuroanatômicas, psicológicas e imunológicas com humanos, representando, com alto valor preditivo, - potencial de novas abordagens terapêuticas para diversas condições em estudos pré-clínicos, sendo atualmente $\mathrm{o}$ modelo experimental mais relevante na determinação da eficácia e segurança em abordagens translacionais ${ }^{5}$. A utilização de primatas em pesquisas é um assunto contencioso devido aos dilemas éticos associados, todavia ainda premente para o alcance de avanços científicos, progressos médicos na descoberta de novos tratamentos para doenças ainda incuráveis e tratamentos mais eficazes para doenças curáveis, fazendo-se necessários ainda em um futuro próximo ${ }^{6}$. Dada a constante preocupação da sociedade acerca de seu uso, conservação e manutenção, as bases legais que normatizam o uso de primatas em pesquisas figuram frequentemente nas agendas políticas nacionais e internacionais, com constantes revisões em suas diretrizes, como ocorrido com a Resolução Normativa $n^{\circ} 28$ de 13/11/2015 do Conselho Nacional de Controle de Experimentação Animal (CONCEA) e a
Diretiva 2010/63/EC da União Europeia, que impõem restrições e limitações mais severas para o uso de primatas em pesquisas científicas.

Não obstante quão bem embasados sejam os argumentos científicos acerca da necessidade do uso de primatas em investigações científicas, deve-se considerar, quando do planejamento experimental, o melhor balanço entre custos e benefícios para todos os sujeitos sencientes envolvidos na pesquisa. Ainda, o rígido cumprimento dos princípios de redução, substituição e refinamento ${ }^{7}$ de primatas constitui aspecto mandatório para garantir resultados experimentais confiáveis, minimizar riscos, reduzir o estresse e promover o bem-estar dos animais.

Especificamente para primatas, um programa de habituação e treinamento para os procedimentos experimentais e manejo consiste em refinamento essencial na redução dos níveis do estresse experimentados pelos animais, provenientes tanto da condição de cativeiro como das manipulações experimentais ${ }^{6}$. Primatas apresentam processos cognitivos intrincados, interações sociais complexas e um repertório comportamental diversificado, sendo suscetíveis à apresentação de desordens comportamentais, psiquiátricas e sofrimento psicológico em resposta ao estresse $^{8}$. Dessa forma, o estabelecimento de relações apropriadas entre pesquisador e primata apresenta indiscutível relevância no contexto da pesquisa, especialmente pela dependência da cooperação do animal para a realização de tarefas comportamentais e cognitivas, havendo a necessidade de uma interação entre o pesquisador e o animal ${ }^{9}$.

envolvimento da equipe de cuidado dos animais é um fator importante, que influencia todo o processo e validade científica dos dados ${ }^{10}$. Interações positivas pesquisador-primata podem melhorar o bem-estar dos animais e aumentar a habilidade dos mesmos em lidar com possíveis fatores estressores ${ }^{11}$. Primatas também tendem a ser sensíveis ao número de pessoas no ambiente, sendo ideal que o treinamento seja realizado com apenas um ou dois pesquisadores. A interação constante dos animais com a mesma equipe permite - reconhecimento de características comportamentais individuais dos animais, que podem influenciar 0 aprendizado devido a preferências por recompensas ou por pesquisadores, uma vez que os primatas também são capazes de reconhecer os humanos, o que pode afetar os resultados dos testes ${ }^{12}$. A boa interação pesquisador-primata tende a atenuar a apresentação de comportamentos aberrantes, dada à redução do estresse associado à manipulação e à interação com o humano, e deve ser buscada no sentido de diminuir a variabilidade dos dados induzida por estresse, aumentar a segurança da equipe de trabalho, reduzindo a probabilidade de lesões por comportamentos agressivos dos animais ${ }^{13}$, além de proporcionar maior conforto e bem-estar para o animal.

Quando da não aplicação de tais medidas de refinamento, a validade dos experimentos pode ser comprometida, já que fatores estressores induzem 
alterações em diversas variáveis fisiológicas ${ }^{14}$, que podem afetar, de forma direta e indireta, os resultados experimentais. Por exemplo, o simples posicionamento em aparatos de contenção para realização de experimentos, como a cadeira para primatas, resulta em restrição de movimentos, o que, para a maior parte dos animais, configura fator estressor ${ }^{15,16}$ e não deve ser utilizada sem treinamento prévio, sob risco de - simples uso do aparato comprometer os resultados dos experimentos. Todavia, apesar de existir um senso comum acerca do reconhecimento da necessidade de treinamento e habituação como refinamento experimental e de todos os benefícios associados, essas técnicas não são tão amplamente aplicadas, devido a fatores que envolvem disponibilidade de pessoal, tempo, falta de preparo, treinamento e escassez de informações detalhadas acerca de como implementá-las ${ }^{17}$.

Quando da implementação de qualquer protocolo de treinamento para primatas em ambiente experimental, é importante considerar as diferenças biológicas, fisiológicas e etológicas da espécie a ser utilizada, que afetam todos os aspectos da vida do animal no ambiente de laboratório ${ }^{18}$, e devem influenciar diretamente decisões acerca do tipo de alimento que deve ser oferecido, bem como as variáveis ambientais e os procedimentos experimentais. No caso dos Sapajus, por exemplo, sabe-se que possuem repertório comportamental variado e são os únicos primatas neotropicais que brincam com objetos que são colocados em seus compartimentos ${ }^{19}$, o que influencia as estratégias de enriquecimento ambiental para animais do gênero. Além disso, macacos do gênero Sapajus exibem típica hierarquia social, com ocorrência de indivíduos dominantes (machos e fêmeas alfa) ${ }^{20}$, e é sabido que os machos alfa são mais suscetíveis à apresentação de respostas comportamentais a fatores estressores $^{21}$.

As técnicas e processos de habituação e treinamento ao uso de aparatos experimentais geralmente são encarados como meios para um fim, e descrições acerca de seus resultados e variações não são frequentes na literatura. Apesar disso, pode-se identificar pelo menos três tipos de abordagens observadas na adaptação das interações entre pesquisador e primata e no treinamento comportamental:

I - A habituação, resultante de estimulação neutra repetitiva, isto é, a simples presença do estímulo (pesquisador), não associada a qualquer reforço ou punição, que com o tempo induz a redução do comportamento de medo, levando, em alguns casos, ao primata ignorar o observador. Poucos estudos descreveram a evolução comportamental de primatas durante o processo de habituação para manejo em cativeiro, havendo mais dados disponíveis referentes à habituação à presença de humanos em ambientes naturais ${ }^{22,23}$. É importante ressaltar que o repertório comportamental de primatas durante tais processos apresenta poucas generalidades, com frequentes apresentações espécie-específicas ${ }^{24}$;
II - A punição negativa e a positiva, que consistem na retirada de um estímulo reforçador (recompensa) para a punição negativa ou a inserção de um estímulo aversivo para a punição positiva, quando há apresentação de um comportamento indesejado ${ }^{10}$. A punição positiva é bastante questionada do ponto de vista ético, uma vez que pode apresentar efeitos psicológicos indesejáveis, caso o arranjo experimental, a intensidade e a duração dos estímulos não sejam adequados $^{25}$. Tais efeitos podem incluir em primatas a apresentação de quadros de ansiedade de forma bastante similar à observada em humanos ${ }^{26}$; e

III - O reforçamento, podendo ser positivo ou negativo, que pode ser descrito como a inserção de uma "consequência" a um determinado comportamento, utilizado de forma a torná-lo mais provável, sendo o reforço positivo a inserção de um estímulo reforçador (recompensa) e o reforço negativo a retirada de um estímulo aversivo. $\bigcirc$ reforçamento negativo, assim como a punição positiva, é uma técnica eticamente questionável, apesar de haver adaptações evitando abordagens que causem dor ou desconforto, podendo ser utilizadas de forma aceitável e com bons resultados ${ }^{27,28}$. Desse modo, o reforçamento positivo consiste na estratégia mais indicada para o treinamento de comportamentos desejáveis em primatas em ambiente experimental. Essa técnica pode auxiliar na melhora da relação pesquisador-primata no que diz respeito ao comportamento do animal ao colaborar em procedimentos que incluem o seu próprio cuidado, de modo a reduzir o estresse e a apresentação de comportamentos anormais, podendo servir ainda como estratégia de enriquecimento ambiental ${ }^{10,29,30}$.

$\bigcirc$ uso de técnicas de reforçamento positivo não necessita da privação prévia de água ou alimentos e demonstra resultados satisfatórios no treino de primatas para a cooperação em testes comportamentais, bem como em procedimentos como restrição, aplicação de injetáveis, manejo e transporte para limpeza e manutenção das gaiolas, diminuindo a chance de danos físicos ou psicológicos aos animais ${ }^{4}$. Tipicamente, as técnicas envolvem o uso de um estímulo reforçador condicionado (também chamado de estímulo-nexo) entre o reforço positivo e o comportamento apresentado, que tem o efeito de acelerar o processo de condicionamento das respostas do animal ${ }^{10}$. Uma técnica muito utilizada é a do clicker, amplamente aplicada para treinamento de primatas em ambiente de laboratório ${ }^{31}$. Resultados positivos no treino para aplicação de injetáveis, saída e retorno voluntário de gaiolas e comportamentos mais complexos foram relatados em diversas espécies, incluindo Callithrix jacchus, Macaca mulatta, Pan troglodytes, Cercocebus atys, entre outros $32,33,34,35,36,37$. Tradicionalmente, o reforço resulta no aumento da frequência de resposta; todavia, a redução do intervalo para a apresentação da resposta (latência) também pode ser apresentada como um resultado da alteração da alocação temporal da resposta como consequência do reforçamento ${ }^{38}$.

No presente estudo, objetivou-se determinar o padrão de aprendizado e as possíveis diferenças inter 
e intraindividuais observadas em treinamento para entrada voluntária e posicionamento de macacos Sapajus apella em aparato experimental - cadeira para primatas - utilizando a técnica de reforçamento positivo, bem como quantificar o tempo necessário para o alcance dos resultados esperados, a fim de fornecer dados que possam nortear diretrizes e parâmetros de promoção do bem-estar animal para instituições que mantêm esses animais em cativeiro ou os utilizem para pesquisa científica.

\section{MATERIAIS E MÉTODOS}

\section{ANIMAIS}

Participaram da pesquisa três macacos-pregos (Sapajus apella) machos, com média de idade estimada de 12 anos, peso médio de 4,300 $\pm 0,99 \mathrm{~kg}$, provenientes da colônia de reprodução do Centro Nacional de Primatas (Ananindeva, estado do Pará, Brasil). No período anterior aos experimentos, todos os indivíduos foram mantidos em grupos de reprodução constituídos por dois machos e dez fêmeas, e alocados em gaiolas duplas de $2,5 \times 2,0 \times 2,5 \mathrm{~m}$ cada, com janela de comunicação que permitia a movimentação livre dos animais em um espaço de 2,5 x 4,0 × 2,5 m.

Os indivíduos selecionados para os experimentos deste estudo não faziam parte do mesmo grupo de reprodução e não participaram de experimentos anteriores. Durante todo o período de realização dos experimentos, os animais ficaram em um galpão, separado do restante da colônia, contendo somente os animais experimentais, alojados lado a lado em gaiolas individuais contendo dois compartimentos de $75 \mathrm{~cm} \times 75 \mathrm{~cm} \times 75 \mathrm{~cm}$ cada, sendo um na parte superior e um na parte inferior da gaiola. $\bigcirc$ acesso entre os compartimentos era feito através de um tubo de passagem feito de aço inoxidável, permitindo ao animal livre trânsito entre os compartimentos superior e inferior. $O$ compartimento inferior continha brinquedos, cordas e aparatos de enriquecimento ambiental que eram trocados diariamente, e o enriquecimento de dieta foi realizado durante todo o período dos experimentos $^{39}$. $\bigcirc$ galpão possuía amplas janelas que proporcionavam iluminação natural, com ciclo claro-escuro regular $(12 / 12 \mathrm{~h})$ durante todo $\mathrm{O}$ ano (latitude $01^{\circ} 21^{\prime} 56^{\prime \prime} \mathrm{S}$; longitude $\left.48^{\circ} 22^{\prime} 20^{\prime \prime}\right)$. Os animais foram submetidos a uma dieta constituída por ração própria para animais de laboratório, ofertada diariamente, associada à oferta de frutas e sucos intercalados quatro vezes por semana, às $12 \mathrm{~h}$, e água ad libitum. Todos os procedimentos foram realizados de acordo com as recomendações e requerimentos éticos da Diretriz 2010/63/EU da União Europeia e sob aprovação da Comissão de Ética para o Uso de Animais do Instituto Evandro Chagas (CEUA/IEC), certificados $n^{\circ} 45 / 2016$ e $n^{\circ} 37 / 2018$.

\section{PROCEDIMENTO EXPERIMENTAL}

Inicialmente, os três animais foram submetidos a sessões diárias individuais de habituação ao convívio com os experimentadores pelo período de dois meses. Dois experimentadores, sempre os mesmos, participaram de todas as sessões durante todo o período de treinamento. As sessões eram realizadas no período da manhã, antes do horário da alimentação dos animais. Ao início da sessão, era realizada a retirada do tubo de comunicação dos compartimentos da gaiola, isolando o animal na parte superior. Em seguida, ocorria a interação dos experimentadores com o animal, por meio do oferecimento de recompensas comestíveis, para criar um contato e para o animal se adaptar à presença do experimentador. $\bigcirc$ experimentador apenas se deslocava para pontos diferentes da gaiola e oferecia as recompensas ao animal. Após um mês de experimentos, foram inseridas as interações ativas, por meio de gestos, oralizações e toques por parte do experimentador, seguidos de recompensas. Cada sessão de habituação tinha duração de $2 \mathrm{~h}$ com cada animal. As recompensas eram variadas e consistiam em cereais, frutas secas e bolachas, e aquelas que os animais demonstrassem preferência eram adotadas para serem utilizadas nas fases posteriores. Todas as recompensas foram previamente avaliadas e liberadas pelo médico veterinário responsável. Após o período de dois meses, os animais demonstraram estar mais habituados à presença dos experimentadores, engajando-se facilmente na interação com os mesmos e estando mais solícitos aos comandos.

Após o período de habituação aos experimentadores, os animais foram submetidos a sessões de treino para posicionamento na cadeira para primatas, com a técnica de condicionamento operante por reforçamento positivo. As sessões foram realizadas por um período de aproximadamente quatro meses, sendo cada animal treinado individualmente, três vezes por semana, totalizando 50 sessões por animal. Os animais eram treinados no período da manhã, em sessões com duração de 20 min. Cada sessão era iniciada com a retirada do acesso do animal ao compartimento inferior da gaiola e o posicionamento do aparato experimental - a cadeira para primatas (Figura 1) - no orifício de encaixe do tubo de passagem do animal. Para o treino da entrada voluntária e posicionamento correto do animal no aparato, foi utilizada a técnica do clicker. Essa técnica consiste no estabelecimento de uma associação ou pareamento entre o som do clicker e a apresentação de uma recompensa, de modo que o som serve como um nexo entre o estímulo não condicionado e a recompensa posteriormente obtida, levando à apresentação do comportamento desejado ${ }^{40}$. Uma vez tornado evidente que o animal compreendeu a associação entre o som e a apresentação da recompensa, o reforçador condicionante deve ser utilizado para sinalizar ao animal quando este apresentar um comportamento desejado, que deverá ser seguido da entrega da recompensa comestível. Posteriormente, o treinamento foi continuado somente com a entrega das recompensas comestíveis aos animais quando da apresentação dos comportamentos desejados. 
A

\section{CADEIRA PARA PRIMATAS}

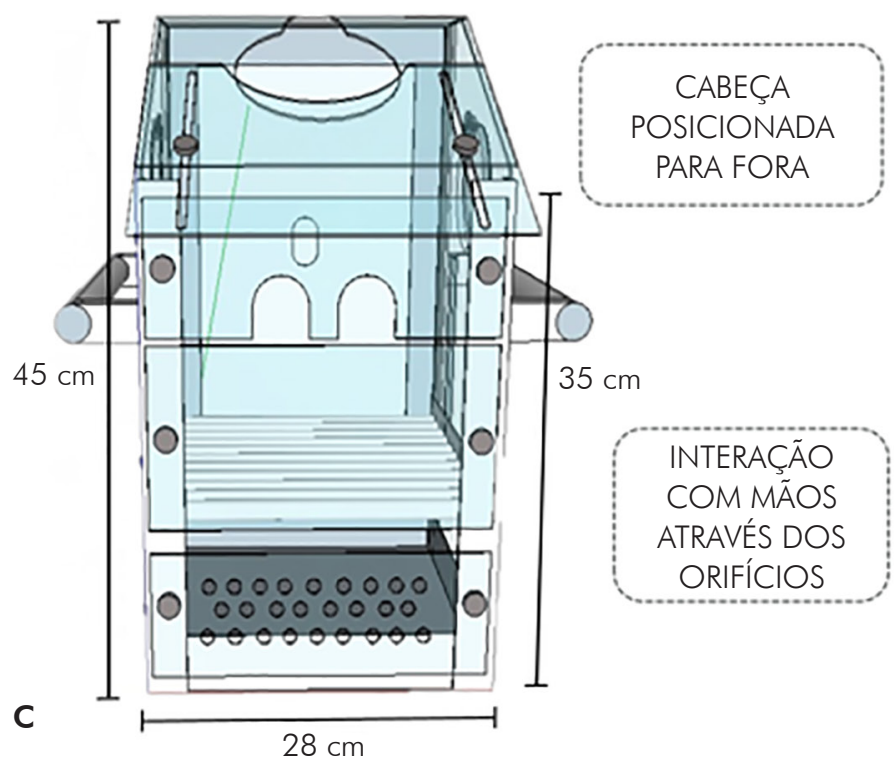

COMPORTAMENTOS DESEJADOS

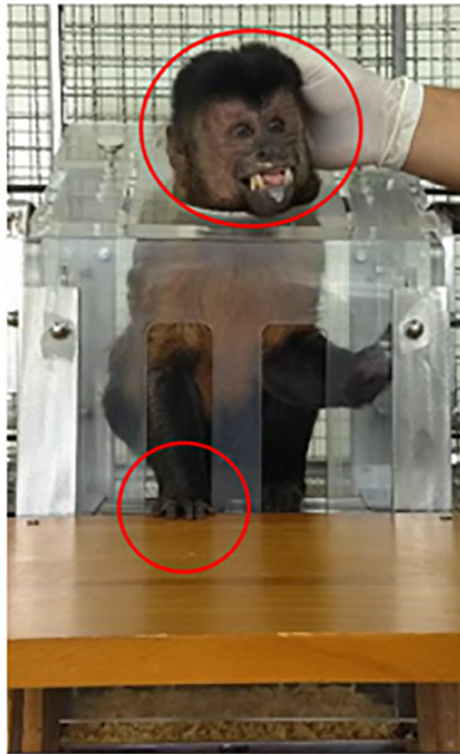

PERMISSIVIDADE AO TOQUE

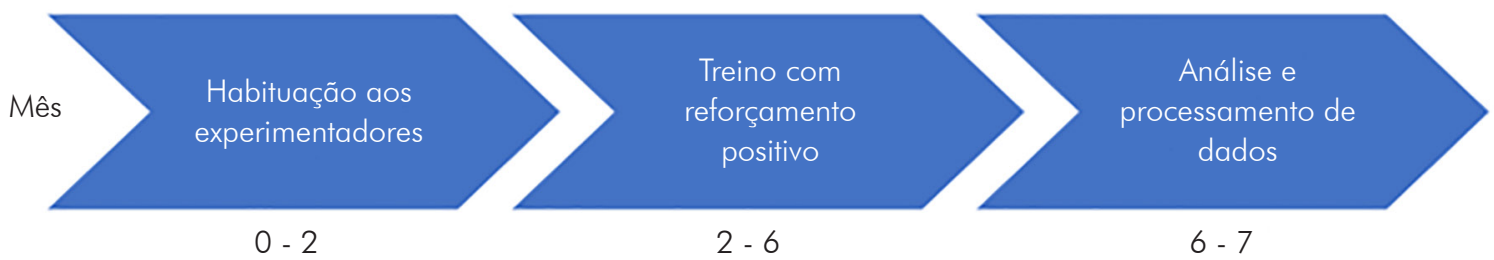

A: Modelo em sketchup da cadeira para primatas adaptada, utilizada no presente estudo; B: Animal posicionado durante a fase de treinamento, apresentando os comportamentos desejados de permissividade ao toque, cabeça e membros superiores devidamente inseridos e posicionados nos orifícios do aparato; C: Linha do tempo experimental.

Figura 1 - Treino para posicionamento do animal na cadeira para primatas

objetivo principal do treinamento consistia na entrada e posicionamento voluntário do animal na cadeira para primatas, com cabeça e membros superiores devidamente posicionados nos orifícios destinados a esse fim (Figura 1), e permitindo a manipulação e interação através de toques por parte dos experimentadores. A fim de garantir uma permanência constante do animal, especialmente no posicionamento da cabeça, além das recompensas sólidas comestíveis era ofertada uma recompensa líquida (suco ou iogurte) posicionada acima do orifício da cabeça, de modo que o animal, para consumi-la, necessitasse estar com a cabeça corretamente posicionada no aparato.

No presente estudo, tomou-se o cuidado para que todas as sessões de treinamento fossem realizadas com os mesmos dois pesquisadores, e que houvesse troca constante das recompensas, para manutenção do enriquecimento e motivação dos animais, bem como a suspensão da sessão de treinamento caso o animal apresentasse sinais de estresse ou agitação. Um treino realizado com reforçamento puramente positivo envolve a não coerção do animal, de modo que o progresso acontece de acordo com a progressão natural do mesmo. A fim de monitorar se o treinamento estava sendo fator indutor de estresse nos animais, durante todo o período dos experimentos, eles foram cuidadosamente observados pela equipe de manejo, veterinários e pesquisadores em seu dia a dia para a identificação de alterações comportamentais indicativas de aumento de estresse e/ou ansiedade e apresentação de comportamentos estereotipados ou agressivos, que eram relatados, quando da sua ocorrência e situação, à equipe de pesquisadores.

A cadeira para primatas foi desenvolvida a partir de modelos já existentes utilizados em outros estudos ${ }^{41}$ e adaptada às especificidades do projeto experimental em questão, assegurando o conforto dos animais durante a restrição parcial para testes futuros. $\bigcirc$ aparato consiste em uma caixa de acrílico $(45 \mathrm{~cm} \times 35 \mathrm{~cm} \times 28 \mathrm{~cm})$, reforçada com barras de aço inoxidável, com aberturas frontais para os braços e superior para a cabeça, dispositivo ajustável para posicionamento da cabeça com restrição parcial e assento regulável de acordo com o tamanho de cada animal. A cadeira era fixada na porta da gaiola, como uma extensão desta, garantindo o fácil acesso do animal durante a sessão de treinamento de aprendizado ao posicionamento correto na cadeira, sendo retirada ao término de cada sessão.

As sessões de treinamento para posicionamento na cadeira para primatas foram gravadas em vídeo para posterior análise da quantidade e duração da apresentação dos parâmetros comportamentais a seguir: 
1 Tempo de toque na cabeça (tempo total da sessão que $\mathrm{o}$ animal permitia $\mathrm{o}$ experimentador tocar em sua cabeça);

2 Tempo com a cabeça para a fora (tempo total da sessão que o animal permanecia com a cabeça posicionada para fora do aparato no local destinado);

3 Toque na cabeça (número de vezes que o animal permitia ser tocado na cabeça);

4 Número de vezes que o animal colocava a cabeça para fora;

5 Tempo de toque no tronco (tempo total da sessão que o animal permitia o experimentador tocar em seu tronco);

6 Número de vezes que o animal permitia ser tocado no tronco;

7 Latência de entrada (tempo que o animal levava para realizar a primeira entrada no aparato, a partir da abertura da porta da gaiola);

8 Número de entradas espontâneas (número de vezes que o animal entrava no aparato sem qualquer tipo de estímulo por parte do experimentador);

9 Tempo de permanência espontânea (tempo total da sessão que o animal permanecia no aparato sem qualquer estímulo por parte do experimentador);

10 Tempo de consumo da recompensa líquida (tempo total que o animal levava para consumir a recompensa líquida);

11 Número de pegadas pela frente (número de vezes que o animal pegava a recompensa pelas aberturas frontais do aparato, posicionando nelas os membros superiores);

12 Número de pegadas com a boca (número de vezes em que o animal pegava a recompensa da mão do experimentador com a boca); e

13 Tempo de permanência na cadeira (tempo total da sessão que o animal permanecia dentro do aparato).

Durante toda a sessão, o animal tinha a liberdade de entrar e sair do aparato de forma voluntária, não havendo restrição ou limitação do mesmo para a manutenção de quaisquer posições ou comportamentos. Somente o estímulo de oferecimento das recompensas era utilizado para a manutenção do posicionamento do animal no aparato. As recompensas sólida e líquida eram oferecidas acima do orifício de posicionamento da cabeça, para a retirada com a boca, podendo o animal fazer a escolha entre os estímulos, e pelos orifícios de posicionamento dos membros superiores, onde eram oferecidas somente recompensas sólidas, para serem recebidas pelo animal com as mãos. As recompensas de preferência dos animais, listadas na fase de habituação, eram utilizadas para as sessões de treinamento, sendo alternadas diariamente. Em um mesmo dia, a mesma recompensa era oferecida para todos os animais.

\section{ANÁLISE DOS DADOS}

Os dados quantitativos foram obtidos por meio das análises manuais dos vídeos e quantificações temporais e/ou de quantidade de vezes na qual um determinado comportamento fora apresentado pelo animal durante a sessão de treinamento, os quais foram organizados em planilhas no Microsoft Excel e analisados por estatística paramétrica utilizando os softwares BioEstat v5.0 e GraphPad Prism. Foram realizados testes de normalidade para a detecção de extremos, seguidos de testes estatísticos para verificar diferenças significativas. Para a verificação do fator aprendizado nos testes, utilizou-se o método de médias móveis, que permite a estimativa de uma curva de aprendizado. Para analisar a evolução comportamental e verificar as diferenças entre os dados obtidos em diferentes janelas temporais ao longo do processo de condicionamento, as médias dos escores obtidos pelos três animais durante as sessões de treinamento na cadeira foram divididas em cinco períodos de dez sessões subsequentes cada. Foi aplicado o teste ANOVA de duas vias para a comparação das médias recebidas pelos animais nos períodos, e foram verificadas as diferenças entre os valores obtidos nas cinco janelas temporais ao longo da curva durante o período de treinamento, denominadas P1, P2, P3, P4 e P5, correspondentes a dez sessões cada. Considerou-se a obtenção de platô na curva de aprendizado quando a curva tendia à saturação, observada por inspeção visual, e correspondente a quando o primeiro valor no início do plano da curva não era excedido por qualquer dos cinco valores seguintes. As curvas de comportamento foram analisadas individualmente, e a análise de variância ANOVA de uma via foi aplicada para a verificação de diferenças interindividuais e diferenças de desempenho entre as janelas temporais nos parâmetros comportamentais observados. $\bigcirc$ intervalo de confiança mínimo adotado para a significância estatística foi de 95\% ( $p<0,05)$.

\section{RESULTADOS}

\section{ANÁLISE DA EVOLUÇÃO COMPORTAMENTAL DOS ANIMAIS}

Os animais experimentais, aqui identificados como M1, M2 e M3, exibiram o comportamento voluntário de entrar e se posicionar na cadeira para primatas em 30 sessões, em média, durante o período de oito semanas. Todos os animais foram capazes de associar o clicker à apresentação dos comportamentos desejados. $O$ treinamento utilizado foi exclusivamente por reforçamento positivo. Houve uma única exceção para o primata $M 1$, que não atendeu ao critério para o posicionamento correto da cabeça no tempo necessário para a permanência no aparato. Todavia, o animal aprendeu a entrar espontaneamente na cadeira para primatas e usar as aberturas frontais para manipulação de objetos e coleta de recompensas comestíveis que, por sua vez, eram ofertadas em posições fora do aparato experimental. 
A análise da evolução comportamental, por meio das médias dos escores obtidos pelos três animais durante as sessões de treinamento, nos períodos $\mathrm{Pl}$ a P5, revelou diferenças estatisticamente significativas para a maioria dos parâmetros comportamentais. Dos 13 parâmetros observados, 12 apresentaram diferenças consideráveis entre os cinco períodos, com aumentos nos escores positivos, com exceção para o parâmetro permissividade ao toque no corpo (Tabela 1).

As curvas de aprendizagem expressas pelas médias móveis demonstraram a obtenção dos melhores escores, ou o início de um platô, por volta da $30^{a}$ sessão para a maior parte dos parâmetros comportamentais analisados (Figura 2).

\section{ANÁLISE DAS DIFERENÇAS INDIVIDUAIS NO PROCESSO DE CONDICIONAMENTO}

Análises interindividuais dos parâmetros comportamentais durante o treinamento na cadeira para primatas demonstraram diferenças significativas entre os sujeitos. As comparações das médias individuais das sessões apresentaram diferenças com relação à permissividade do toque da cabeça, posicionamento da cabeça no aparato, latência para entrada e permanência no aparelho, tempo para consumo da recompensa líquida e correto posicionamento e interação pelas aberturas frontais do aparelho. Os resultados estão ilustrados na figura 3 .

Foram observadas diferenças interindividuais nas curvas de aprendizagem expressas para cada animal. Inicialmente, o estabelecimento do platô das curvas de aprendizagem foi diferente entre $M 1, M 2$ e M3 lo platô foi considerado como estabelecido quando o maior valor para um dado parâmetro não foi superado nas cinco sessões seguintes), com o sujeito mais rápido dominando o comportamento básico em aproximadamente 20 sessões, e o sujeito mais lento fazendo-o em cerca de 37 sessões para atender ao mesmo critério. Ml apresentou diferenças significativas de desempenho com relação a $M 2$ e M3, tendo apresentado clara evitação para - posicionamento da cabeça, todavia com melhor desempenho no número de entradas espontâneas no aparato experimental e posicionamento das mãos, enquanto $M 2$ apresentou maiores escores no tempo de permanência espontânea, mas aumento na latência de entrada nas fases iniciais. Para alguns dos parâmetros comportamentais analisados, não foi constatado estabelecimento de platô, como tempo de toque na cabeça e tempo de consumo da recompensa líquida (Figura 4).

Tabela 1 - Dados comportamentais dos primatas durante os períodos de treinamento para posicionamento na cadeira Períodos de treinamento

Parâmetros comportamentais

$\mathrm{P} 1$

$32.8 \pm 6.2^{\ddagger}$

$37.0 \pm 10.0^{\ddagger}$

$4.2 \pm 0.6$

$1.7 \pm 0.3^{\ddagger}$ fora

5 Tempo de toque no tronco

6 Toque no tronco

7 Latência de entrada

8 Número de entradas espontâneas

9 Tempo de permanência espontânea

10 Tempo de recompensa líquida

11 Número de pegadas pela frente

12 Número de pegadas com a boca

13 Tempo de permanência na cadeira

$161.9 \pm 0.9 \neq$

$14.2 \pm 1.3^{\ddagger}$

$38.5 \pm 3.0^{\ddagger}$

$$
6.5 \pm 0.6
$$

$152.3 \pm 13.3$

P2

P3

P4

P5

$36.3 \pm 9.1^{\ddagger}$
$129.4+34.4^{\ddagger}$

$72.4 \pm 17.4^{\ddagger}$

$394.5 \pm 32.2^{\ddagger}$

$725.6 \pm 55.5^{\ddagger}$

$393.5 \pm 26.3^{\ddagger}$

$545.7 \pm 18.7 \ddagger$

$635.4 \pm 11.0^{\ddagger}$

$$
4.2 \pm 0.9
$$

$2.9 \pm 0.4^{*}$

$5.2 \pm 0.6$

$5.6 \pm 0.7$

$7.8 \pm 1.3$

$11.0 \pm 1.2^{+}$

$6.9 \pm 0.5$

$6.9 \pm 0.4$

$8.1 \pm 0.6$

$\begin{array}{ccccc}152.3 \pm 13.3 & 207.1 \pm 24.5 & 148.8 \pm 17.4 & 167.2 \pm 9.5 & 163.3 \pm 10.3 \\ 95.2 \pm 13.4^{\ddagger} & 85.0 \pm 16.4^{\ddagger} & 288.4 \pm 17.6^{\ddagger} & 404.4 \pm 17.5^{\ddagger} & 428.9 \pm 29.2^{\ddagger} \\ 16.7 \pm 1.7^{\ddagger} & 18.7 \pm 1.6^{\ddagger} & 13.8 \pm 1.8^{\ddagger} & 7.7 \pm 0.8 & 6.2 \pm 0.6 \\ 3.5 \pm 0.6 & 6.0 \pm 1.1 & 6.5 \pm 1.1 & 6.5 \pm 0.6 & 9.4 \pm 1.6^{\dagger} \\ 715.7 \pm 56.1^{*} & 799.6 \pm 34.3^{*} & 636.8 \pm 35.7 & 722.8 \pm 14.1 & 879.1 \pm 36.6^{\ddagger}\end{array}$

As médias foram obtidas para cada período de dez sessões comportamentais denominados P1-P5. ANOVA de duas vias; ${ }^{*} \mathrm{p}<0,05 ;{ }^{\dagger} \mathrm{p}<0,01$; $\ddagger \mathrm{p}<0,001$. 

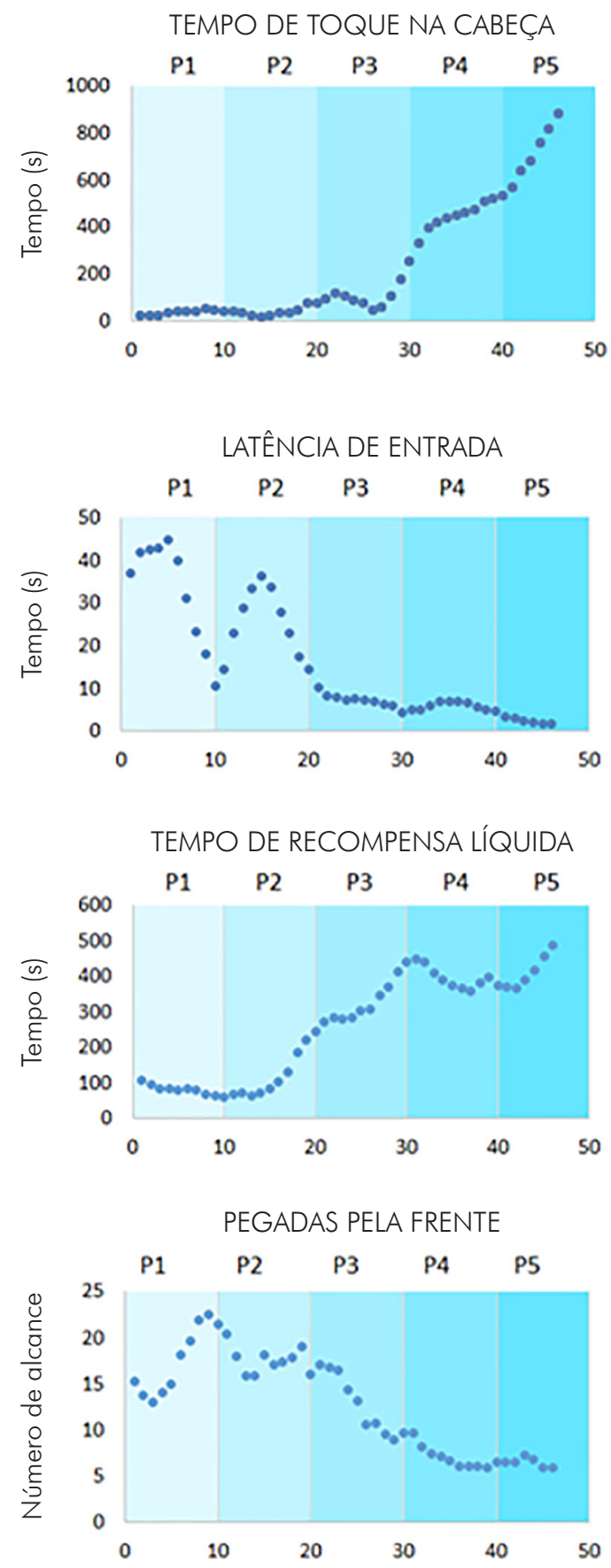
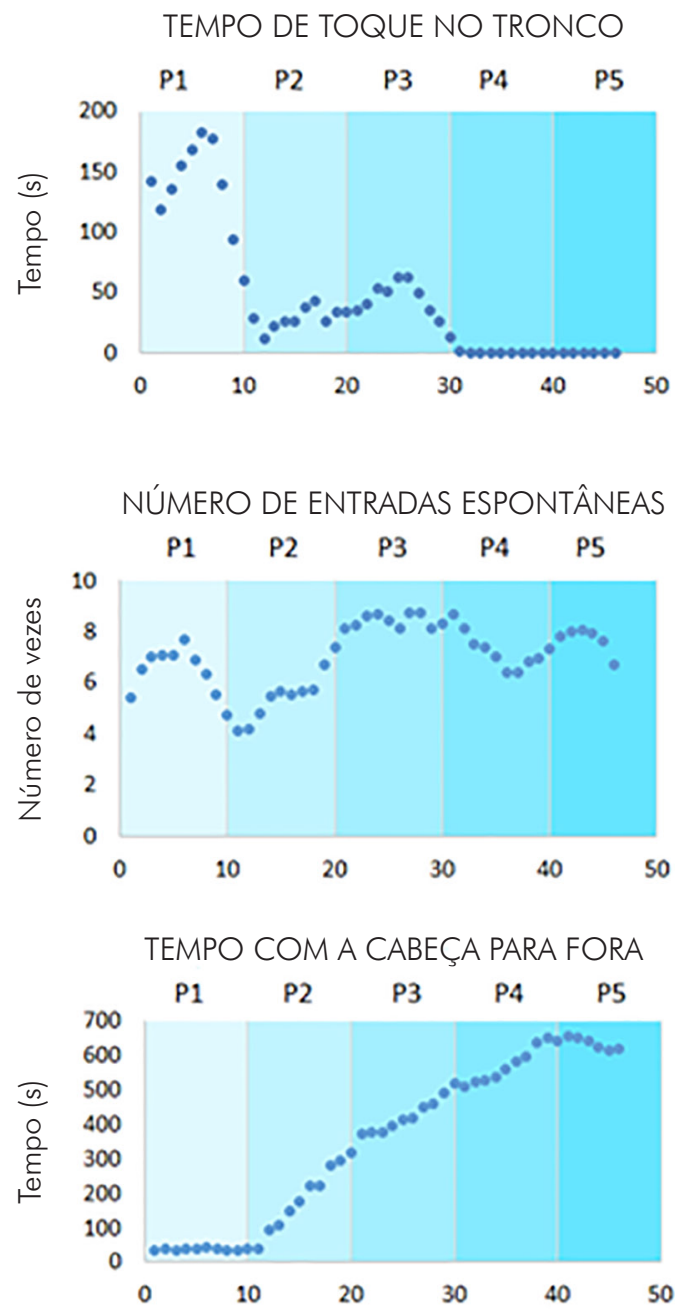

TEMPO DE PERMANÊNCIA NA CADEIRA

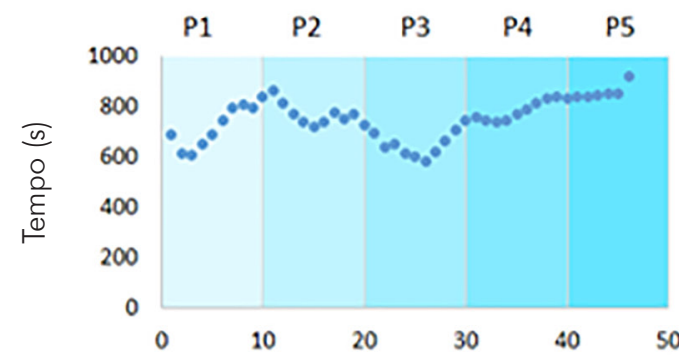

Figura 2 - Curvas de aprendizagem estimadas por médias móveis do grupo de animais durante os períodos de treinamento

\section{DISCUSSÃO}

O objetivo do presente estudo foi analisar os padrões e variações interindividuais e intraindividuais de aprendizado durante treinamento para entrada voluntária e posicionamento em cadeira para primatas, utilizando técnica de reforçamento positivo em macacos. Os resultados obtidos indicam que o método clicker e o condicionamento por reforço positivo foram capazes de induzir o aumento da apresentação dos comportamentos desejáveis de entrada e posicionamento na cadeira para primatas nos macacos Sapajus apella testados. Durante o período de treinamento, os animais foram capazes de reconhecer rapidamente a conexão entre o clicker e as recompensas, e desenvolveram disposição em colaborar com os procedimentos a fim de receber as recompensas oferecidas. Todavia, no presente estudo, não foi realizada a coleta de dados nas fases anteriores ao treinamento na cadeira para verificar as diferenças individuais nos tempos de assimilação de cada animal entre o clicker e a recompensa. Todos os sujeitos apresentaram comportamento de entrada espontânea no aparato no decorrer das 50 sessões de treinamento analisadas. 

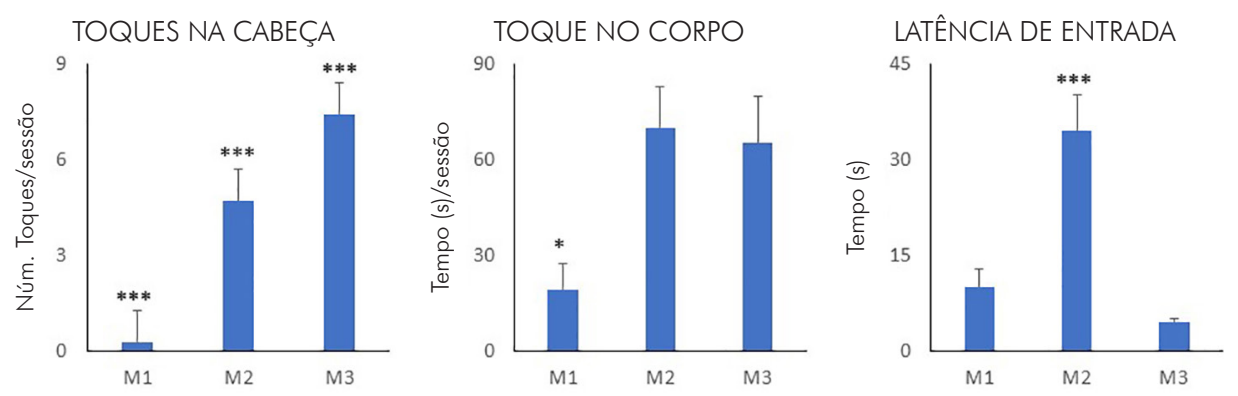

TEMPO DE PERMANÊNCIA

ESPONTÂNEA
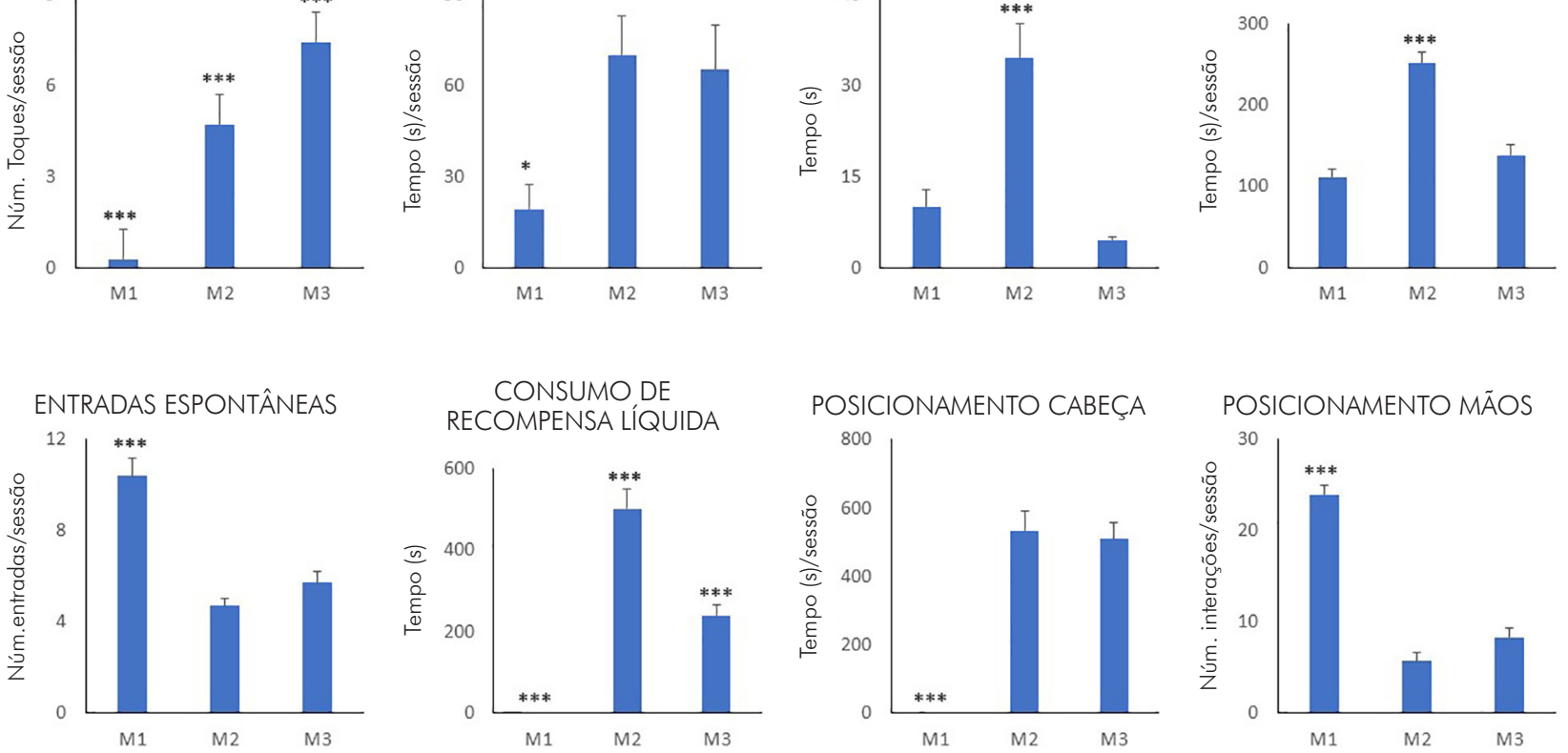

ANOVA de duas vias, teste a priori de Bonferroni; ${ }^{*} p<0,05$; $^{* * *} \mathrm{p}<0,0001$.

Figura 3 - Representação gráfica das médias individuais obtidas nos parâmetros comportamentais analisados durante 0 período de treinamento

A
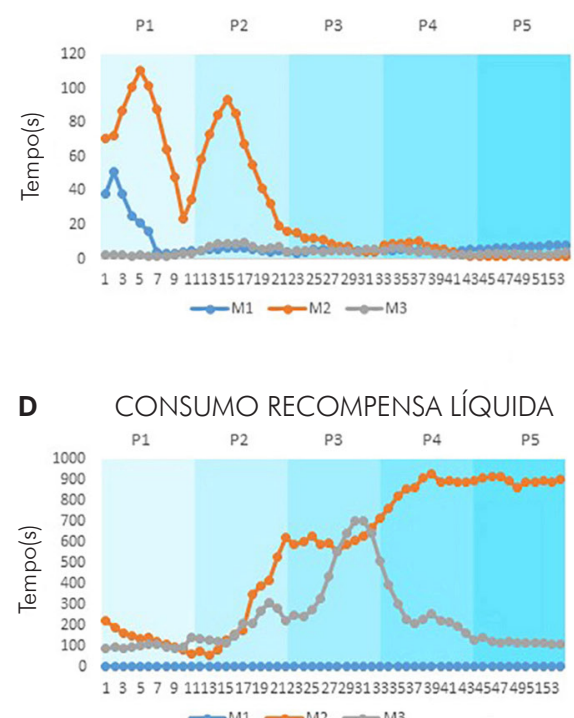

B TEMPO DE PERMANÊNCIA ESPONTÂNEA C

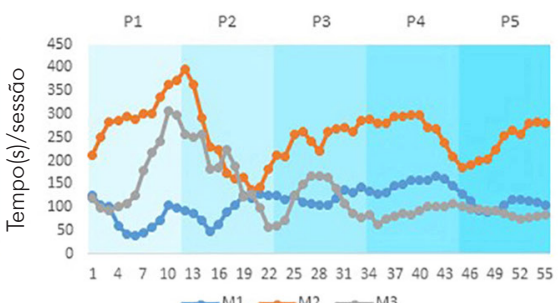

$\rightarrow \mathrm{M} 1 \rightarrow \mathrm{M} 2 \rightarrow \mathrm{MB}$
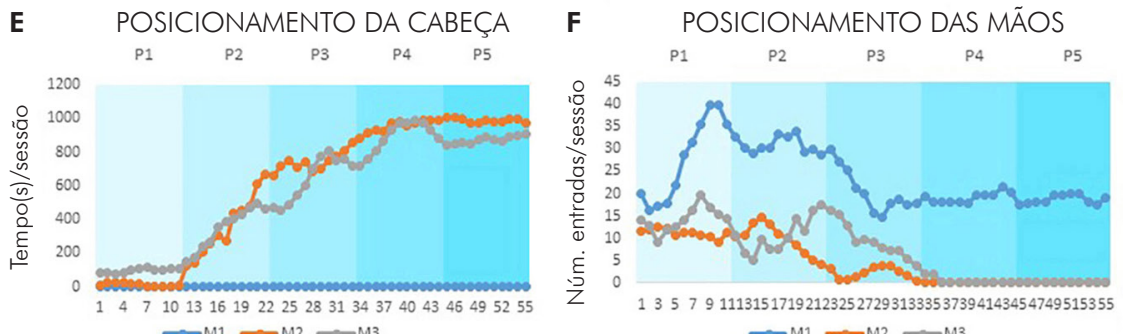

Figura 4 - Curvas de aprendizado individuais estimadas por médias móveis para cada animal durante os períodos de treinamento

processo de aprendizagem apresentou variabilidade entre os animais, e a melhora dos resultados obtidos não seguiu uma trajetória linear para diversas variáveis. De um modo geral, a amostra exibiu resultados que demonstram curvas de aprendizagem com aumento progressivo da frequência e diminuição da latência de apresentação dos comportamentos desejados, todavia com diferentes padrões de aprendizagem observados para cada parâmetro comportamental por macaco, o que pode resultar de preferências individuais. A análise de variância entre as médias obtidas nos períodos de P1 a P5 demonstraram aumento na permissividade dos animais para toques na cabeça, tempo com a cabeça posicionada corretamente no aparato (para fora) e tempo para consumo da recompensa líquida, bem como redução progressiva na latência de entrada espontânea do animal no aparato, dados que em conjunto indicam, de forma indireta, uma possível redução da ansiedade experimentada pelo animal quando da restrição dos movimentos e da cabeça durante o posicionamento do pescoço no orifício do aparato. 
As curvas de aprendizagem, que podem ser definidas como representações gráficas do desempenho em função do tempo de prática, não demonstraram ter alcançado um platô para algumas variáveis, demonstrando, inclusive para alguns comportamentos, um padrão crescente ao início, seguido de fase decrescente. Estudos dedicados à análise de padrões comportamentais demonstraram que curvas de aprendizagem podem ser altamente variáveis ${ }^{42,43}$. Não obstante, reconhece-se que a amostra ainda não alcançou a fase final de aprendizado para todos os comportamentos desejados, que, de acordo com predições baseadas em outros estudos que aplicaram o treinamento por reforço positivo para $\circ$ aprendizado de tarefas motoras em primatas, caracteriza-se pela apresentação de um platô percebido visualmente no final das curvas de aprendizagem, com o primeiro valor não sendo excedido pelos escores das cinco sessões subsequentes ${ }^{44,45}$.

Como para alguns parâmetros comportamentais observou-se uma variação de desempenho ao longo da curva de aprendizado, acredita-se que variáveis como propriedade do treino, fatores motivacionais (como a ausência de privação alimentar anterior às sessões), tempo de coleta de dados, complexidade dos comportamentos pretendidos e outras relacionadas à configuração dos experimentos podem ter influenciado nessa variabilidade, bem como fatores individuais relacionados à dinâmica comportamental dos animais em seus grupos sociais, anteriores ao período de experimentos. Todavia, os resultados do período de aprendizagem foram considerados satisfatórios, especialmente dada a complexidade da tarefa empregada do ponto de vista do ethos dos animais. $\mathrm{O}$ treinamento por reforço positivo requer tempo para se adquirir estabilização da curva de aprendizagem, o que, de certa forma, reduz a praticidade da abordagem por conta do tempo para aprendizado mais prolongado quando comparado com outras técnicas; em alguns cenários experimentais, sua implementação pode representar um desafio devido a limites temporais de execução de projetos de pesquisa. Obviamente, o tempo e a duração do processo de habituação devem variar de acordo com a complexidade do procedimento aprendido e de sua proximidade com o ethos do animal, bem como a frequência de treinamento ${ }^{35}$.

Estudos anteriores aplicando o treino por reforço positivo para a aprendizagem de testes motores em Macaca fascicularis observaram o alcance do platô de aprendizagem após três meses de treinamento diário (três a cinco vezes por semana), com um mínimo de 70 sessões de testes, para o alcance dos resultados nos animais com melhor taxa de aprendizado ${ }^{45}$. Outros trabalhos demonstraram resultados mais rápidos na aquisição dos comportamentos desejados, utilizando combinação de reforçamento positivo e reforçamento negativo em Macaca mulatta ${ }^{30}$. Todavia, é importante ressaltar a impossibilidade de comparação desses resultados com os do presente estudo, dadas as diferenças entre as espécies com repertórios comportamentais diversificados e complexos, bem como configurações experimentais distintas. Ainda, o uso de reforço negativo, quando aplicado, deve ser planejado com cautela para que não atue como um fator estressor, comprometendo a validade de resultados dependentes de outras variáveis fisiológicas e comportamentais em questão.

Apesar de não terem sido realizadas análises fisiológicas relacionadas ao estresse, é importante notar que, durante todo o período do experimento, não foram detectadas evidências físicas ou comportamentais indicativas de estresse nos animais experimentais. Os mesmos foram cuidadosamente observados para a apresentação de sinais como alopecia, comportamentos estereotipados, agressividade, hiper ou hipoatividade, dentre outros, não tendo sido registrado qualquer sinal relevante indicativo de estresse ou ansiedade. Estudos apontam que $\mathrm{o}$ treino por reforço positivo apresenta efeito de redução das respostas fisiológicas ao estresse em animais experimentais. Evidências obtidas por meio do treino por reforçamento positivo em Callithrix jacchus demonstraram que os animais treinados apresentaram redução das respostas comportamentais a fatores estressores quando comparados com animais que não receberam 0 treinamento ${ }^{46}$; e estudos com amostras sanguíneas de animais cativos demonstraram que variáveis fisiológicas indicativas de estresse foram significativamente afetadas pelo treinamento com reforço positivo, com redução do total de leucócitos, neutrófilos segmentados e níveis de glicose em animais que receberam treinamento para a realização de procedimento anestésico, quando comparados com animais que passaram pelos mesmos procedimentos involuntariamente com o uso de métodos tradicionais ${ }^{47}$.

Para algumas das variáveis observadas, houve redução de desempenho dos animais entre os períodos P2 e P3. Esse dado não consiste em um fator preocupante, uma vez que a regressão é considerada como um evento normal durante o processo de treinamento, podendo variar de acordo com características individuais ou do processo de treinamento ${ }^{48}$. A variabilidade individual no processo de aprendizagem foi significativa para quase todos os parâmetros analisados. Ml representou a menor taxa de aprendizagem em diversos parâmetros, com escores significativamente inferiores a M2 e M3, enquanto M2 apresentou um desenvolvimento satisfatório, observado desde o início das sessões de treinamento. Considerando que nenhum dos animais havia participado de experimentos anteriores, que, no período anterior aos experimentos, todos os indivíduos foram mantidos em condições similares de interação social e com humanos, e que variações individuais em taxas de aprendizagem são comuns em qualquer amostra, infere-se que as diferenças interindividuais aqui observadas possam ser apenas um reflexo da variação dentro dos padrões de normalidade.

De fato, os testes de normalidade aplicados aos dados garantiram que não houvesse dados aberrantes. Entretanto, é importante considerar que primatas possuem repertório comportamental variado, decorrente de processamentos psicológicos complexos, e o conhecimento do repertório comportamental 
característico da espécie em questão é relevante na interpretação dos resultados. Em espécies que estabelecem hierarquia social, por exemplo, é sabido que os machos alfa são mais suscetíveis à apresentação de respostas comportamentais a fatores estressores ${ }^{21}$. Sabe-se ainda que os macacos do gênero Sapajus exibem típica hierarquia social, com ocorrência de indivíduos dominantes (machos e fêmeas alfa) ${ }^{20}$. Neste estudo, M1 era o espécime de constituição corpórea mais robusta, quando comparado a $M 2$ e $M 3$, e exibia padrões de comportamento caracteristicamente dominantes (e.g. vocalizações mais elevadas, movimentos mais bruscos, postura mais elevada). Anteriormente aos experimentos, os animais encontravam-se alojados em grupos hierárquicos diferentes, e não foi realizada uma análise de seus papéis nesses grupos quando da seleção dos mesmos para este estudo. Se esses fatores são responsáveis pelas diferenças nos padrões de aprendizagem aqui observados, é uma questão que demanda investigações mais detalhadas.

Por fim, ressalta-se que este estudo apresenta limitações que devem ser consideradas. $\bigcirc$ pequeno número de animais e o curto período analisado estabelecem limites do ponto de vista estatístico, devido ao baixo poder da amostra. Ainda, fontes de vieses experimentais, como a impossibilidade de aplicação de delineamento cego ou randomização de amostras, podem impactar os resultados, juntamente com variáveis comportamentais e psicológicas resultantes da interação pesquisador-primata. Todavia, tratando-se de estudo de viabilidade de técnica de refinamento experimental, sua relevância deve ser considerada em relação aos possíveis impactos positivos que podem advir do treinamento. Tais efeitos podem repercutir sobre a confiabilidade dos resultados experimentais dos experimentos consequentes ao treinamento, bem como sobre os pontos de vista éticos associados ao mesmo.

\section{CONCLUSÃO}

Os resultados obtidos sugerem a eficácia da implementação de treinamento por reforço positivo na indução de comportamentos desejáveis nos animais testados e contribuem para o aprendizado sobre testes comportamentais em primatas. $\bigcirc$ tempo necessário para a aquisição de platô de aprendizado foi superior ao observado na aplicação de técnicas que combinam reforçamento positivo e negativo, todavia não comparáveis por serem experimentos realizados com outras espécies. $\bigcirc$ reforçamento positivo pode afetar positivamente a natureza das relações humano-primata no ambiente de laboratório, constituindo, assim, uma importante técnica de refinamento experimental. Dessa forma, recomenda-se a implementação de diretrizes para a ampla adoção de tais medidas nos protocolos experimentais. Contudo, essas medidas devem considerar as características espécie-específicas, bem como a necessidade de esforços experimentais para a determinação das técnicas mais adequadas, duração das sessões, e discussão acerca do impacto do treinamento no ambiente e resultados experimentais em estudos com primatas.

\section{AGRADECIMENTOS}

Aos técnicos Obadias dos Reis Silva e Sheila Tetsume Makiama, pelo auxílio no manejo e treinamento dos animais experimentais.

\section{APOIO FINANCEIRO}

- presente estudo recebeu financiamento do Programa de Investigação e Inovação Horizonte 2020 da União Europeia, sob o contrato n. ${ }^{\circ} 767092$.

\section{CONFLITOS DE INTERESSES}

Os autores declaram que não há conflitos de interesses.

\section{CONTRIBUIÇÃO DOS AUTORES}

Todos os autores contribuíram para a idealização do estudo, análise e/ou interpretação dos dados e escrita deste manuscrito, aprovando sua publicação. Estes se declaram responsáveis pelo conteúdo, garantindo sua acurácia e integridade.

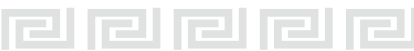

\section{REFERÊNCIAS}

1 Desmond T, Laule G. Use of positive reinforcement training in the management of species for reproduction. Zoo Biol. 1994;13(5):471-7.

2 Laule G, Desmond T. Positive reinforcement training as an enrichment strategy. In: Shepherdson D, Mellen J, Hutchins $M$, editors. Second nature: environmental enrichment for captive animals. Washington: Smithsonian Institution Press; 1998. p. 302-12.

3 Ramirez K. Animal training: successful animal management through positive reinforcement. Chicago: Shedd Aquarium; 1999.
4 Prescott MJ, Buchanan-Smith HM. Training nonhuman primates using positive reinforcement techniques. J Appl Anim Welf Sci. 2003;6(3):157-61.

5 Arnason G, Clausen J. On balance: weighing harms and benefits in fundamental neurological research using nonhuman primates. Med Health Care Philos. 2016 Jun; 19(2):229-37.

6 Prescott MJ. Ethics of primate use. Adv Sci Res. $2010 ; 5(1): 11-22$.

7 Russell WMS, Burch RL. The principles of humane experimental technique. London: Methuen; 1959. 
8 Shively CA, Willard SL. Behavioral and neurobiological characteristics of social stress versus depression in nonhuman primates. Exp Neurol. 2012 Jan;233(1):87-94.

9 Prescott MJ, Brown VJ, Flecknell PA, Gaffan D, Garrod K, Lemon RN, et al. Refinement of the use of food and fluid control as motivational tools for macaques used in behavioural neuroscience research: report of a Working Group of the NC3Rs. J Neurosci Methods. 2010 Nov; 193(2):167-88.

10 Westlund K. Training laboratory primates-benefits and techniques. Primate Biol. 2015;2(1):119-32.

11 Baker KC. Benefits of positive human interaction for socially-housed chimpanzees. Anim Welf. 2004 May; 13(2):239-45.

12 Waitt C, Buchanan-Smith HM, Morris K. The effects of caretaker-primate relationships on primates in the laboratory. J Appl Anim Welf Sci. 2002;5(4):309-19.

13 Rennie AE, Buchanan-Smith HM. Refinement of the use of non-human primates in scientific research. Part I: the influence of humans. Anim Welf. 2006; 15(3):203- 13.

14 Lapin BA, Grozdik TE, Klots IN. Blood glucose levels in rhesus monkeys (Macaca mulatta) and cynomolgus macaques (Macaca fascicularis) under moderate stress and after recovery. Bull Exp Biol Med. 2013 Feb;154(4):497-500.

15 Shirasaki Y, Yoshioka N, Kanazawa K, Maekawa T, Horikawa T, Hayashi T. Effect of physical restraint on glucose tolerance in cynomolgus monkeys. J Med Primatol. 2013 Jun;42(3):165-8.

16 Ruys JD, Mendoza SP, Capitanio JP, Mason WA. Behavioral and physiological adaptation to repeated chair restraint in rhesus macaques. Physiol Behav. 2004 Sep;82(2-3):205-13.

17 Prescott MJ, Buchanan-Smith HM. Training laboratory-housed non-human primates, part I: a UK survey. Anim Welf. 2007;16(1):21-36.

18 Wolfensohn S, Honess P. Handbook of primate husbandry and welfare. New York: John Wiley \& Sons; 2008.

19 Emmons LH, Feer F. Neotropical rainforest mammals: a field guide. Chicago: University of Chicago; 1997.

20 Morton FB, Lee PC, Buchanan-Smith HM, Brosnan SF, Thierry B, Paukner A, et al. Personality structure in brown capuchin monkeys (Sapajus apella): comparisons with chimpanzees (Pan troglodytes), orangutans (Pongo spp.), and rhesus macaques (Macaca mulatta). J Comp Psychol. 2013;127(3):282-98.

21 Sapolsky RM. The influence of social hierarchy on primate health. Science. 2005 Apr;308(5722):648-52.
22 van Krunkelsven E, Dupain J, van Elsacker L, Verheyen R. Habituation of bonobos (Pan paniscus): first reactions to the presence of observers and the evolution of response over time. Folia Primatol. 1999 Nov-Dec;70(6):365-8.

23 Blom A, Cipolletta C, Brunsting AMH, Prins HHT. Behavioral responses of gorillas to habituation in the Dzanga-Ndoki National Park, Central African Republic. Int J Primatol. 2004 Feb;25(1):179-96.

24 Williamson EA, Feistner AT. Habituating primates: processes, techniques, variables and ethics. In: Setchell JM, Curtis DJ, editors. Field and laboratory methods in primatology: a practical guide. Cambridge: Cambridge University Press; 2003. p. 25-39.

25 Mayer PCM, Gongora MAN. Duas formulações comportamentais de punição: definição, explicação e algumas implicações. Acta Comport. $2011 ; 19(4): 47-63$.

26 Coleman K, Pierre PJ. Assessing anxiety in nonhuman primates. ILAR J. 2014;55(2):333-46.

27 Wergård EM, Temrin $H$, Forkman B, Spångberg $M$, Fredlund $H$, Westlund $K$. Training pair-housed Rhesus macaques (Macaca mulatta) using a combination of negative and positive reinforcement. Behav Processes. 2015 Apr;1 13:51-9.

28 McLean AN. The positive aspects of correct negative reinforcement. Anthrozoös. 2005 Apr; 18(3):245-54.

29 Coleman K, Maier A. The use of positive reinforcement training to reduce stereotypic behavior in rhesus macaques. Appl Anim Behav Sci. 2010 May; 124(3-4): 142-8.

30 McMillan JL, Perlman JE, Galvan A, Wichmann T, Bloomsmith MA. Refining the pole-and-collar method of restraint: emphasizing the use of positive training techniques with rhesus macaques (Macaca mulatta). J Am Assoc Lab Anim Sci. 2014 Jan;53(1):61-8.

31 Westlund K. Training laboratory primates-benefits and techniques. Primate Biol. 2015;2(1):119-32.

32 McKinley J, Buchanan-Smith HM, Bassett L, Morris K. Training common marmosets (Callithrix jacchus) to cooperate during routine laboratory procedures: ease of training and time investment. J Appl Anim Welf Sci. 2003;6(3):209-20.

33 Coleman K, Pranger L, Maier A, Lambeth SP, Perlman JE, Thiele E, at al. Training rhesus macaques for venipuncture using positive reinforcement techniques: a comparison with chimpanzees. J Am Assoc Lab Anim Sci. 2008 Jan;47(1):37-41.

34 Videan EN, Fritz J, Murphy J, Borman R, Smith HF, Howell S. Training captive chimpanzees to cooperate for an anesthetic injection. Lab Anim. 2005 May;34(5):43-8. 
35 Fernström $A L$, Fredlund $H$, Spångberg $M$, Westlund K. Positive reinforcement training in rhesus macaques-training progress as a result of training frequency. Am J Primatol. 2009 May; $71(5): 373-9$.

36 Veeder CL, Bloomsmith MA, McMillan JL, Perlman JE, Martin AL. Positive reinforcement training to enhance the voluntary movement of group-housed sooty mangabeys (Cercocebus atys atys). J Am Assoc Lab Anim Sci. 2009 Mar;48(2): 192-5.

37 Rogge J, Sherenco K, Mailing R, Thiele E, Schapiro S, Lambeth $S$, et al. Establishing a positive reinforcement training program in Neotropical primates: a comparison of squirrel and owl monkeys. Am J Primatol. 2011 Sep;73 Suppl 1:84.

38 Baum WM. Rethinking reinforcement: allocation, induction, and contingency. J Exp Anal Behav. 2012 Jan;97(1):101-24.

39 Boere V. Environmental enrichment for neotropical primates in captivity. Cienc Rural. 2001 May-Jun;31 (3):543-51.

40 Schapiro SJ, Bloomsmith MA, Laule GE. Positive reinforcement training as a technique to alter nonhuman primate behavior: quantitative assessments of effectiveness. J Appl Anim Welf Sci. 2003;6(3): 175-87.

41 Nassi JJ, Lomber SG, Born RT. Corticocortical feedback contributes to surround suppression in V1 of the alert primate. J Neurosci. 2013 May;33(19):8504-17.
42 Newell KM, Liu YT, Mayer-Kress G. Time scales in motor learning and development. Psychol Rev. 2001 Jan; 108(1):57-82.

43 Schmidt RA, Lee TD, Winstein CJ, Wulf G, Zelaznik $\mathrm{HN}$. Motor control and learning: a behavioral emphasis. 6th ed. Champaign (IL): Human Kinetics; 2018.

44 Chatagny P, Badoud S, Kaeser M, Gindrat AD, Savidan J, Fregosi $M$, et al. Distinction between hand dominance and hand preference in primates: a behavioral investigation of manual dexterity in nonhuman primates (macaques) and human subjects. Brain Behav. 2013 Sep;3(5):575-95.

45 Kaeser M, Chatagny P, Gindrat AD, Savidan J, Badoud S, Fregosi $M$, at al. Variability of manual dexterity performance in non-human primates (Macaca fascicularis). Int J Comp Psychol. $2014 ; 27(2): 295-325$.

46 Bassett L, Buchanan-Smith HM, McKinley J, Smith TE. Effects of training on stress-related behavior of the common marmoset (Callithrix jacchus) in relation to coping with routine husbandry procedures. J Appl Anim Welf Sci. 2003;6(3):221-33.

47 Lambeth SP, Hau J, Perlman JE, Martino M, Schapiro SJ. Positive reinforcement training affects hematologic and serum chemistry values in captive chimpanzees (Pan troglodytes). Am J Primatol. 2006 Mar;68(3):245-56.

48 Warren JM, Sinha MM. Interactions between learning sets in monkeys. J Genet Psychol. 1959 Sep;95(1):19-25. 